\title{
A Two-Stage Monte Carlo Approach for Optimization of Bimetallic Nanostructures
}

\author{
Rossen Mikhov ${ }^{1}$, Vladimir Myasnichenko ${ }^{2}$, Leoneed Kirilov ${ }^{1}$, \\ Nickolay Sdobnyakov ${ }^{2}$, Pavel Matrenin ${ }^{3}$, Denis Sokolov ${ }^{2}$, and Stefka Fidanova ${ }^{1}$ \\ ${ }^{1}$ Bulgarian Academy of Sciences \\ Acad. G. Bonchev Str., bl. 2 \\ 1113 Sofia, Bulgaria \\ Email: 1_kirilov_8@abv.bg
}

\begin{abstract}
In this paper we propose a two-stage lattice Monte Carlo approach for optimization of bimetallic nanoalloys: simulated annealing on a larger lattice, followed by simulated diffusion. Both algorithms are fairly similar in structure, but their combination was found to give significantly better solutions than simulated annealing alone. We also discuss how to tune the parameters of the algorithms so that they work together optimally.
\end{abstract}

\section{INTRODUCTION}

' I 'HE fundamental and practical significance of studying the structural characteristics and transformations in nanoparticles and nanosized heterostructures is associated with the wide prospects for their use in various fields of nanotechnology. For example, they may serve as nanocontacts/nanowires, as sensors, or as catalysts. In this context, the search for stable configurations is a very important research problem [1], [2], [3]. A configuration is stable when its potential energy is minimal. This is a global optimization problem: traditional numerical methods are impractical because they need huge amounts of computational resources [4]. Therefore, the global minimum has to be approximated using time-efficient optimization strategies (metaheuristics).

A lot of methods are available for the prediction of nanoparticle structures [5]. For example, metal nanowires are studied in [6] by means of canonical Monte Carlo simulations and embedded atom potentials, demonstrating some advantages of Monte Carlo simulations over molecular dynamics simulations. In [7], grand and semigrand canonical global optimization approaches are presented, using basin-hopping with an acceptance criterion based on the local contribution of each potential energy minimum to the (semi)grand potential. Details regarding the implementation of the basin-hopping method are also given in relation to

This work was partially funded by Russian Federation of Basic Research, project number 20-37-70007, by the Ministry of Science and Higher Education of the Russian Federation in the framework of the State Program in the Field of the Research Activity, project number 0817-2020-0007, by the National Scientific Program "Information and Communication Technologies for a Single Digital Market in Science, Education and Security (ICTinSES)", Ministry of Education and Science Bulgaria, and by the Bulgarian NSF under the grant DFNI-DN 02/10.
Monte Carlo moves that change the system size. The basin-hopping Monte Carlo algorithm was modified to determine a global minimum structure in $\mathrm{Ag}$ and AgPd nanoclusters [8]. For a pure metallic silver nanocluster, the newly developed quadratic basin-hopping Monte Carlo algorithm is more efficient than the standard basin-hopping Monte Carlo algorithm. For a bimetallic AgPd nanocluster, the new algorithm succeeds in finding the global minimum structure even though the standard algorithm fails. It is important that such approach as the formation energy machine learning model [9] can be used to predict the stable metal element distribution in the nanoparticles via Monte Carlo simulations. In [10], Monte Carlo sampling for pure random selection of sample points is used. It can be useful when implementing the so-called surrogate models, which can be a suitable replacement for complex simulation models in applications.

\section{THE BASIC ALGORITHMS}

Our method performs the optimization on a lattice, combining two Monte Carlo algorithms. The energy of the system is given by the multi-particle tight-binding potential of Cleri-Rosato [11], having the following form:

$$
\begin{gathered}
E=\sum_{i} \sum_{j \neq i} E_{i j, a b}-\sum_{i} \sqrt{\sum_{j \neq i} B_{i j, a b}} \\
E_{i j, a b}=A_{a b} \exp \left(-p_{a b}\left(\frac{r_{i j}}{r_{0, a b}}-1\right)\right) \\
B_{i j, a b}=\xi_{a b}^{2} \exp \left(-2 q_{a b}\left(\frac{r_{i j}}{r_{0, a b}}-1\right)\right)
\end{gathered}
$$

where $i$ ranges over all atoms; $j$ ranges over all atoms other than $i$ but within distance $R_{\text {cut }}$ from $i ; a$ and $b$ represent the species of the atoms $i$ and $j ; E_{i j, a b}$ and $B_{i j, a b}$ are the repulsive and binding components of the potential due to the atom pair $(i, j) ; r_{i j}$ is the distance between the atoms; $r_{0, a b}, A_{a b}, p_{a b}, \xi_{a b}, q_{a b}$ are constants particular to the chemical elements under considera- 
tion. We use a value for $R_{\text {cut }}$ corresponding to five coordination spheres, beyond which the interaction is assumed to be zero.

\section{A. The Wide-Lattice Monte Carlo Algorithm}

The first algorithm, which we will call the "wide-lattice" Monte Carlo, is specified in [12]. It starts by placing the atoms at random on a lattice several times larger than the total number of atoms. At each iteration, one atom and one neighboring empty node are chosen at random. If the potential energy would decrease by the atom moving into the empty node, the jump is performed unconditionally. Otherwise, the jump may still be performed, with a probability given as:

$$
P=\exp (-\Delta E / k T)
$$

where $\Delta E$ is the energy difference of the configurations and $T$ is the current temperature of the system. The iteration ends either with or without a jump.

The temperature is set high at the beginning, and then gradually decreases as the algorithm proceeds. We use a linear formula for the cooling, subtracting a small amount once every several thousand iterations. The algorithm ends when the temperature reaches $1 \mathrm{~K}$.

The appropriate initial temperature strongly depends on the size and type of lattice used, as well as on the size and chemical composition of the nanostructure [13], therefore it is best determined experimentally.

Due to its simplicity and its particular form, this algorithm lends itself to a highly optimized computer implementation. It can run for billions of iterations within minutes on a standard personal computer.

\section{B. The Diffusion Algorithm}

The second algorithm, which we will call the "diffusion," is specified in [14]. It runs on a lattice filled with atoms of two different kinds, plus a small number of empty nodes $(\sim 4$ for a 200-atom structure). At each iteration, one empty node is chosen at random, and the iteration always ends with a jump of a neighboring atom into that empty node. Which atom jumps is determined by calculating (4) separately for each candidate, and picking a random number in the interval from zero to the sum of all $P$ s.

Note that the term neighboring atom is defined here as being within a radius of three coordination spheres. This is different from the wide-lattice Monte Carlo algorithm above, where nodes are neighbors only within one coordination sphere. This difference is due to the scarcity of the empty nodes during diffusion, and we have verified experimentally that three coordination spheres seem to be optimal for this purpose.

Temperature is managed similarly to the wide-lattice Monte Carlo algorithm.

The running time, while slower than the wide-lattice Monte Carlo, is still on the order of millions of iterations per minute.

\section{THE COMBINED METHOD}

The starting point of this research is the observation that combining the two algorithms above may produce better solutions than just a single-staged approach. The proposed combined method has the following steps:

Step 1: Parameter tuning. Repeatedly run the wide-lattice Monte Carlo algorithm from random initial configurations, for $N / 10$ ( 40 million $)$ iterations at each trial, to determine the optimal initial temperature, cooling speed, and scaling factors (along each of the $x, y$, and $z$ axes) for the lattice. Due to the lower number of iterations used, each trial completes quickly to save time for the more important following steps.

Step 2: Shape fixing. Using the best values from Step 1 for the initial temperature and scale factors, repeatedly run the wide-lattice Monte Carlo algorithm afresh from random initial configurations, with $1 / 10$ th the cooling speed, i.e. for $N$ ( 400 million) iterations at each trial. However, set up the nanoalloy to have $\alpha(\sim 2)$ extra atoms of each type. The goal of this step is to obtain the advantageous geometric shapes appropriate for the nanoparticle (3D), film (2D) or wire (1D) under consideration on the given lattice. The resulting configurations also have a somewhat low-energy ordering of the atoms, to be further improved by diffusion.

Step 3: Diffusion. From each resulting configuration, delete all empty nodes, then convert $\alpha$ atoms of each type into empty nodes, to use as vacancies during diffusion. This is the same number of extra atoms added in Step 2, but the converted atoms are selected randomly. Run the diffusion algorithm once per configuration, for $N / 2$ ( 200 million $)$ iterations at each trial.

After all Monte Carlo simulation is finished, following existing practice [12], relaxation with molecular dynamics (MD) may be used to further improve the energy of the system before selecting the best solution as the final result.

\section{VERIFICATION}

We do a number of tests to verify that the proposed method is a significant improvement over simpler approaches.

All trials are performed for a 200-atom $\mathrm{AuAg}$ nanoparticle (gold and silver in 1:1 proportion), on a 309-node lattice with a twinned bi-pyramid shape. One example configuration of this particle is illustrated on Fig. 1. It is expected that the results also hold for other chemical compositions and other lattices (we have observed this in our preliminary testing). Instead of relaxation with MD, all comparisons of final solutions below are done after applying an additional round of scaling of the lattice (with separate factors for the $x, y$, and $z$ axes).

In Fig. 2, the combined method (Steps $1+2+3$ ) is compared against running only the wide-lattice Monte Carlo algorithm (Steps 1+2, but for a larger number of iterations). 


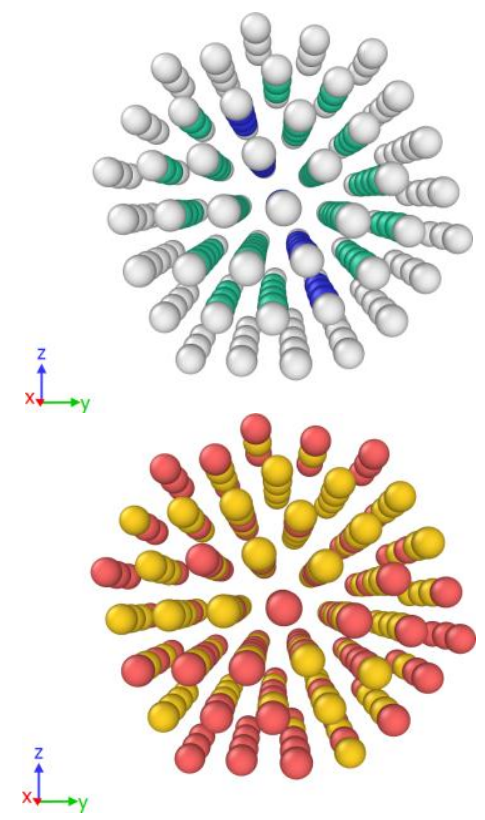

Fig. 1 One example configuration of Au100Ag100 (top: blue - fcc atoms, green - hcp atoms, grey - unknown atoms; bottom: yellow - Au atoms, red - Ag atoms)

The number of iterations is chosen such that the total wall-clock running time is the same in both cases. The combined method gives clearly better solutions, which is the main result of this research.

In Fig. 3, tests verify that the parameter tuning approach of Step 1 is sound. In other words, that the optimal initial temperature determined at the higher cooling speed of Step 1 ( $N / 10$ iterations) is still optimal when the algorithm is run

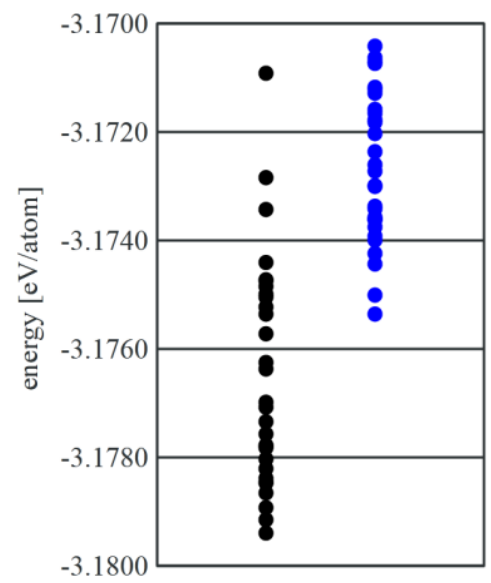

\begin{tabular}{|l|l|}
\hline left side (black) dots: 30 trials & \multicolumn{1}{|c|}{ right side (blue) dots: 30 trials } \\
\hline combined method & wide-lattice algorithm only \\
Step 1: 40 million iterations & Step 1: 40 million iterations \\
Step 2: 400 million iterations & Step 2: 4 billion iterations \\
Step 3: 170 million iterations & (so that total running time is the same) \\
\hline
\end{tabular}

Fig. 2 Comparing the combined method (Steps $1+2+3$ ) against using only the wide-lattice simulated annealing algorithm (Steps 1+2)

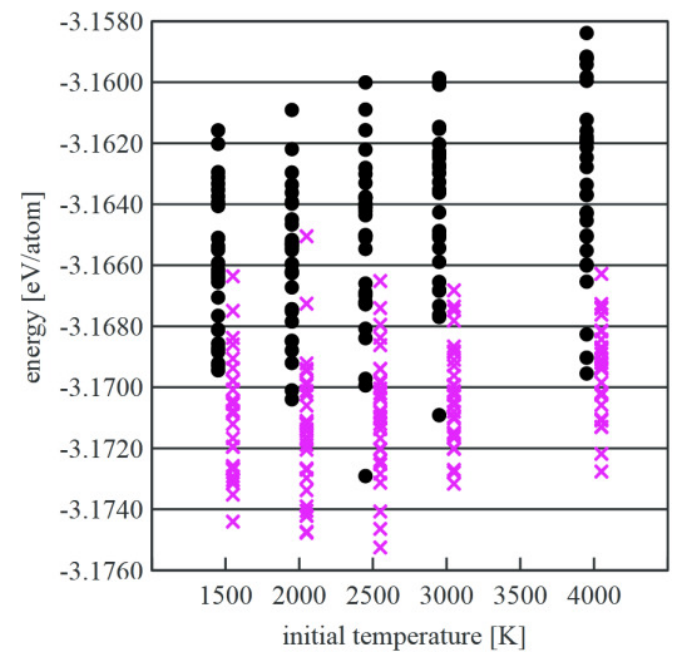

\begin{tabular}{|c|l|}
\hline \multicolumn{2}{|c|}{ for each initial temperature: } \\
\hline left side (black dots): 30 trials & right side (pink crosses): 30 trials \\
\hline fast cooling (40 million iterations) & slow cooling (400 million iterations) \\
\hline
\end{tabular}

Fig. 3 Comparing the optimal initial temperature for fast cooling (such as in Step 1) versus slow cooling (such as in Step 2). The tried temperatures are the same for both cases but the crosses are shown slightly to the right of the dots for clarity.

at the much lower cooling speed of Step 2 ( $N$ iterations). From the figure, it can be read out that the initial temperature giving best results is $2500 \mathrm{~K}$, the same in both cases.

We note that for this lattice, the differences between initial temperatures seem to be small, to the point that any temperature from the chosen range may be adequate. With larger lattices and more atoms, however, the influence of the initial temperature is more dramatic [13], and in that case the advantage of knowing the best initial temperature before running the main algorithm may also be more substantial.

The last series of tests evaluate whether the parameter tuning approach of Step 1 is advantageous. That is, whether the solutions by the full combined method are better than if we only ran Steps $2+3$.

In Fig. 4, comparing the left (black) column with the middle (red) column, it can be seen that there is not much difference between the full combined method and a variant omitting Step 1 but running Step 2 for 10\% more iterations (and since the best initial temperature is assumed unknown, from a random temperature in the range of 1000-4000 K). This corresponds to the fact that the influence of the initial temperature is small, as was already observed in Fig. 3. It remains open whether choosing the initial temperature at random is adequate for larger numbers of atoms, where the temperature effects are amplified.

In Fig. 4, the right (green) column shows the case of an alternative way of determining the lattice scaling factors to be applied before Step 3: instead of performing the scaling after Step 1 and using that for Steps 2+3, omit Step 1 and perform the scaling after Step 2. Comparing these solutions to the left (black) column, we see that the alternative ap- 


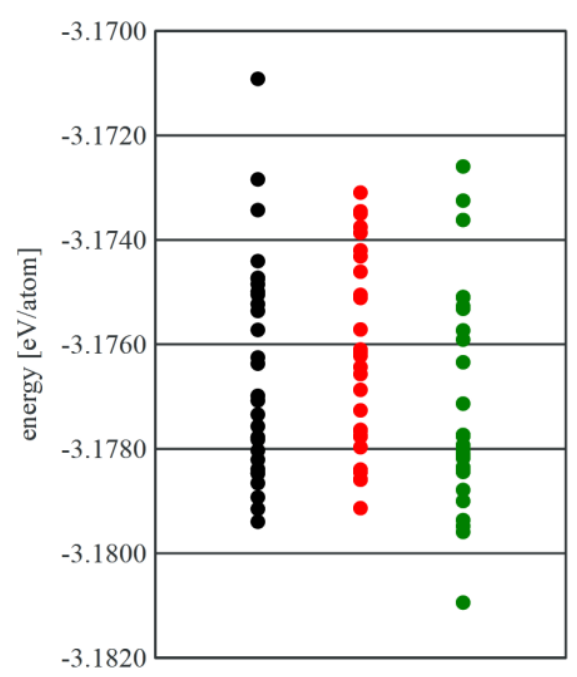

\begin{tabular}{|l|l|l|}
\hline \multicolumn{1}{|c|}{$\begin{array}{c}\text { left side (black) } \\
\text { dots: } 30 \text { trials }\end{array}$} & \multicolumn{1}{c|}{$\begin{array}{c}\text { middle (red) } \\
\text { dots: 30 trials }\end{array}$} & \multicolumn{1}{c|}{$\begin{array}{c}\text { right side (green) } \\
\text { dots: 30 trials }\end{array}$} \\
\hline full combined method & alternative 1 & alternative 2 \\
Step 1: 40 million & random initial & scaling of the lattice \\
iterations & temperature & after Step 2 \\
Step 2: 400 million & Step 2: 440 million & Step 2: 440 million \\
iterations & iterations & iterations \\
Step 3: 170 million & Step 3: 170 million & Step 3: 170 million \\
iterations & iterations & iterations \\
\hline
\end{tabular}

Fig. 4 Influence on the final results of using Step 1 to tune the parameters: the initial temperature (comparing left vs. middle columns), and the lattice scaling factor (comparing left vs. right columns)

proach is not substantially different. If anything, it may even look like the original (black) approach is slightly worse, but this cannot be determined from our data - the test design allows only confirming or failing to confirm a potential advantage, not a disadvantage. (The alternative (green) trials run Step 2 for $10 \%$ more iterations while still starting from the known best initial temperature, which gives them an unfair start.)

\section{V.CONCLUSION}

We have proposed a lattice Monte Carlo method for optimization of bimetallic nanoalloys, combining two previous algorithms: a wide-lattice simulated annealing algorithm and a simulated diffusion algorithm. We have verified that the combined method gives significantly better solutions than using only wide-lattice simulated annealing. We have discussed several ways to tune the algorithm parameters, proposing one particular approach (namely, with an additional tuning step), and verifying its soundness. As regards the alternative parameter tuning approaches investigated, they were found to be equally good to the proposed one on the relatively small lattice on which the tests were performed.

There are other important parameters to be tuned that we have not discussed here. In particular, better managing the temperature during diffusion is something that, in our expe- rience, becomes much more important when a large number of atoms are involved. Further research will be needed to determine the optimal strategy for particle sizes where this becomes relevant. It is also worth mentioning that appropriate size effects at the nanoscale region should be taken into account, in particular, at the interface between components [15], [16].

\section{REFERENCES}

[1] D. J. Wales and J. P. K. Doye, "Global optimization by basin-hopping and the lowest energy structures of Lennard-Jones clusters containing up to 110 atoms," J. Phys. Chem. A., vol. 101, no. 28, pp. 5111-5116, July 1997.

[2] X. Wu and Y. Sun, "Stable structures and potential energy surface of the metallic clusters: $\mathrm{Ni}, \mathrm{Cu}, \mathrm{Ag}, \mathrm{Au}, \mathrm{Pd}$, and Pt," J. Nanopart Res., vol. 19, art. no. 201, July 2017.

[3] K. Michaelian, N. Rendón, and I. L. Garzón, "Structure and energetics of Ni, Ag, and Au nanoclusters," Phys. Rev. B, vol. 60, no. 3, pp. 2000-2010, July 1999.

[4] J. P. K. Doye, "Physical perspectives on the global optimization of atomic clusters," in Global Optimization. Nonconvex Optimization and Its Applications, vol. 85, J. D. Pintér, Ed. Boston, MA: Springer, 2006, pp. 103-139.

[5] S. B. Gelfand and S. K. Mitter, "Metropolis-type annealing algorithms for global optimization in $\{\mathrm{R}\}^{\wedge} \mathrm{d}$," SIAM J. Control Optim., vol. 31, no. 1 , pp. 111-131, 1993.

[6] M. C. Giménez and W. Schmicker, "Monte Carlo simulation of nanowires of different metals and two-metal alloys," J. Chem. Phys., vol. 134, pp. 064707-1-064707-6, Febr. 2011.

[7] F. Calvo, D. Schebarchov, and D. J. Wales, "Grand and semigrand canonical basin-hopping," J. Chem. Theory Comput., vol. 12, no. 2, pp. 902-909, Dec. 2015.

[8] H. G. Kim, S. K. Choi, and H.M. Lee, "New algorithm in the basin hopping Monte Carlo to find the global minimum structure of unary and binary metallic nanoclusters," J. Chem. Phys., vol. 128, no. 14, pp.144702-1-144702-4, Apr. 2008.

[9] C. Chen, Y. Zuo, W. Ye, et al., "Critical review of machine learning of energy materials," Adv. Energy Mater., vol. 10, no. 8, 1903242-1-1903242-36, Jan. 2020.

[10] S. Balduin, F. Oest, M. Blank-Babazadeh, A. Nieße, and S. Lehnhoff, "Tool-assisted surrogate selection for simulation models in energy systems," in Proc. 2019 FedCSIS, pp. 185-192.

[11] F. Cleri and V. Rosato, "Tight-binding potentials for transition metals and alloys," Phys. Rev. B, vol. 48, no. 1, pp. 22-33, July 1993.

[12] V. Myasnichenko, N. Sdobnyakov, L. Kirilov, R. Mikhov, and S. Fidanova, "Structural instability of gold and bimetallic nanowires using Monte Carlo simulation," in Recent Advances in Computational Optimization: Results of the Workshop on Computational Optimization and Numerical Search and Optimization 2018, S. Fidanova, Ed. Springer, 2020, pp. 133-145.

[13] R. Mikhov, V. Myasnichenko, S. Fidanova, L. Kirilov, and N. Sdobnyakov, "Influence of the temperature on simulated annealing method for metal nanoparticle structures optimization," in Advanced Computing in Industrial Mathematics: 13th Annu. Meet. Bulg. Sect. of SIAM, Dec. 2018, Sofia, Bulgaria, Springer, to be published.

[14] V. Myasnichenko, R. Mikhov, L. Kirilov, N. Sdobnykov, D. Sokolov, and S. Fidanova, "Simulation of diffusion processes in bimetallic nanofilms," in Advanced Computing in Industrial Mathematics: 14th Annu. Meet. Bulg. Sect. of SIAM, Dec. 2019, Sofia, Bulgaria, Springer, submitted for publication.

[15] V. M. Samsonov, N. Yu. Sdobnyakov, A. G. Bembel, D. N. Sokolov, and N. V. Novozhilov, "Size dependence of the melting temperature of metallic films: two possible scenarios," J. Nano-Electron. Phys., vol. 5, no. 4, pp. 04005-1-04005-3, Dec. 2013.

[16] V. M. Samsonov, N. Yu. Sdobnyakov, A. G. Bembel, D. N. Sokolov, and N. V. Novozhilov, "Thermodynamic approach to the size dependence of the melting temperatures of films," Bull. Russ. Acad. Sci. Phys., vol. 78, no. 8, pp. 733-736, Sept. 2014. 